\title{
TANGENTIAL INJECTION TO A SUPERSONIC FLOW ON A BLUNTED NOSE
}

\author{
P. V. Chuvakhov, I. V. Egorov, I. V. Ezhov, I. V. Novikov, \\ and E. B. Vasilevskiy
}

Central Aerohydrodynamic Institute (TsAGI)

1 Zhukovsky Str., Zhukovsky, Moscow Region 140180, Russia

\begin{abstract}
The flow pattern and the heat flux to a body surface at a tangential gas injecting have been investigated. The cooling air was injected to a flow through the tangential axisymmetric slot on the spherically blunted cylinder. The experiments were conducted at $\mathrm{M}_{\infty}=6, \operatorname{Re}_{\infty, R_{w}}$ $=0.76 \cdot 10^{6}$, angle of attack $\alpha=0^{\circ}-30^{\circ}$, and the slot width $h_{k} / R_{w}=0$ 0.021 . The mass rate of the injecting gas was $G^{*}=g_{j} /\left(\pi \rho_{\infty} u_{\infty w}^{2}\right)=0$ 0.16. It has been shown that maximum of the heat flux toward the sphere surface can be sufficiently decreased. Numerical investigations have been carried out using the solution of the Navier-Stokes equations for axisymmetric two-dimensional (2D) viscous compressible unsteady flows at $\alpha=0$.
\end{abstract}

\section{INTRODUCTION}

To gain high a lift-to-drag ratio at a high-speed aircraft, a small bluntness radius is needed for fuselage nosetips, wing leading edges, and other projected elements. Small bluntness is also needed for operating efficiency of inlet of a supersonic air-breathing jet engine. In some cases, low temperature of the vehicle surface is required (e.g., for mounting optical window used by photo devices).

However, with high levels of stagnation temperature and pressure behind the normal shock along with small bluntness radius of aircraft elements, the heat flux is so high that reliable reusable thermal protection cannot be implemented by traditional methods, even using best materials.

Decreasing of heat loads towards the vehicle surface is possible by means of coolant injecting through the permeable surface or using special devices. The detailed analysis showed that the most effective coolants (providing minimal weight and volume of the coolant system) are water, glycerol, ammonia, etc. [1-4].

However, in many cases where gas is available aboard (hydrogen, nitrogen, etc.), it is reasonable to implement the vehicle surface cooling via gas injecting.

(C) The authors, published by EDP Sciences. This is an Open Access article distributed under the terms of the Creative Commons Attribution License 4.0 (http://creativecommons.org/licenses/by/4.0/). 
In the absence of local zones of increased pressure on the wall (e.g., shock wave incidence on the protected surface), the lowest weight cost of the coolant matter injecting is attained by injecting coolant through a microporous material $[5,6]$. But hydraulic properties of microporous material are not stable in operating time and they vary from sample to sample. This is caused, in particular, by the presence of the condensed particles in a coolant and its supply system.

In real-time use of cooling via gas injecting through perforation, injected trickles penetrate into free flow across the boundary layer, even at the smallest size of holes to be manufactured at present time. For nose elements of streamlined bodies (where the boundary layer is generally thin), the penetration of trickles into the outer flow is observed most of the time. This requires very high mass flow rate of the injecting matter [7].

An alteration of hydraulic properties of injecting devices during operation is possible to prevent by injecting through a slot, including the cases with dust and moisture in the coolant and its supply system. This is conditioned by a large width of the slot (tenths of millimeter) in comparison with micropore diameter (microns and tenths of micron), and also because slot channels are almost straight. In $[8,9]$, it was shown that it is possible to cool the lateral surface of a streamlined body using low mass rates of coolant. Tangential injecting through a slot could be more effective in comparison with porous injecting in the case of flat shock wave incidence on a vehicle surface or in the dusted flow $[10,11]$. The present authors do not know any works on flow and heat transfer study in the case of gas injecting through axisymmetric slot placed on the front surface of blunted nose.

This work represents the results of experimental and numerical study of flow and heat transfer on the spherical bluntness surface of a longitudinally streamlined cylinder when air is injecting through a tangential axisymmetric slot. The outlet section of the slot nozzle was located at a small distance $S^{*}=S / R_{w}=0.23$ (the central angle $\psi=13^{\circ}$ ) from the model critical point. It is shown that even at the substantial angle of attack of $30^{\circ}$, it is possible to essentially reduce the maximum of the heat flux towards the model surface using low mass rates of the injecting matter.

The study was carried out in the TsAGI wind tunnel operating in Ludwig scheme at the free flow Mach number $\mathrm{M}_{\infty}=6$, total pressure $p_{0}=30$ bar, and stagnation temperature $T_{0}=710 \mathrm{~K}$. In the experiments, pressure of the injecting gas, the slot width, and the angle of attack were varied.

The case of zero angle of attack was investigated numerically. The $2 \mathrm{D}$ axisymmetric Navier-Stokes equations were solved using the implicit second-order total variation diminishing (TVD) scheme. It is shown that the computed flow fields are in qualitative agreement with the experimental data.

The similar investigations were performed on the sharp wedge model [12-14]. 


\section{EXPERIMENTAL EQUIPMENT}

\subsection{Wind Tunnel}

The study was carried out in the TsAGI wind tunnel operating in Ludwig scheme at the free flow Mach number $\mathrm{M}_{\infty}=6$, total pressure $p_{0}=30$ bar, and stagnation temperature $T_{0}=710 \mathrm{~K}$. Pressure of the injecting gas, the slot width and the angle of attack have been varied in the experiments.

The high-pressure channel of the wind tunnel with the inner diameter of $70 \mathrm{~mm}$ and length 6 or $12 \mathrm{~m}$ is equipped by an external ohmic heater which provides gas heating to $T_{0}=800 \mathrm{~K}$ (Fig. $1[15]$ ). The test section diameter is $2 R_{n}=0.5 \mathrm{~m}$. Duration of the steady-state flow is $\tau=23$ or $46 \mathrm{~ms}$.

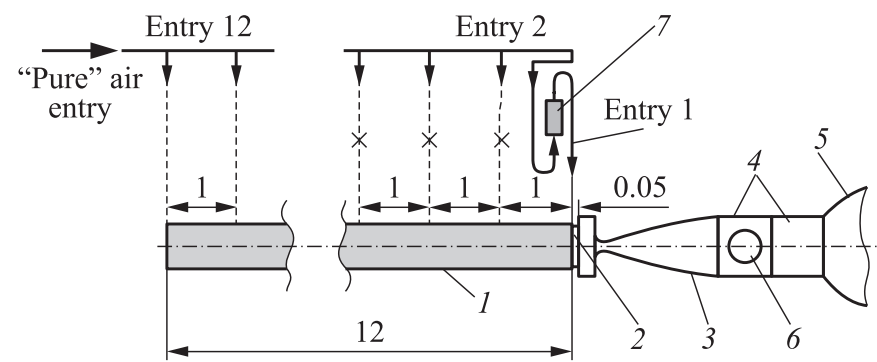

Figure 1 The wind tunnel UT-1: 1 - duct; 2 - diaphragm; 3 - nozzle; 4 test section; 5 - exhaust chamber; 6 - optical window; and 7 - mixing device. Dimensions are in meters

\subsection{Model}

The model is a spherically blunted cylinder (Fig. 2). The cooling gas (air) is supplied through a central tube 4 . The tangential axisymmetric slot is implemented between a flat "cap" and the sphere surface $(A)$. The slot width $h$ can be changed by rotating of the stem "cap" with very small thread in the central tube.

Because of the sphere curvature, the slot width in the exit section $h_{e}$ is larger than in the minimal entrance section $h_{k}$. Thus, the ratio of the areas in critical and exit sections of the slot is $F_{k} / F_{e}=\left(d_{k} h_{k}\right) /\left(d_{e} h_{e}\right)$. When the minimal slot width is $h_{k}=0.2 \mathrm{~mm}$, the rated Mach number at the slot exit is $\mathrm{M}=2.56$ and at the slot width $h_{k}=0.8 \mathrm{~mm}$, the Mach number is $\mathrm{M}=2.04$. In all cases, the injecting flow is supersonic that guarantees a uniform distribution of coolant in the azimuthal direction.

In the experiments, gas was supplied to the model by means of the fastoperating system. The cross section of each element of the system essentially exceeds the minimal cross section of the slot. Therefore, the gas velocity in the 


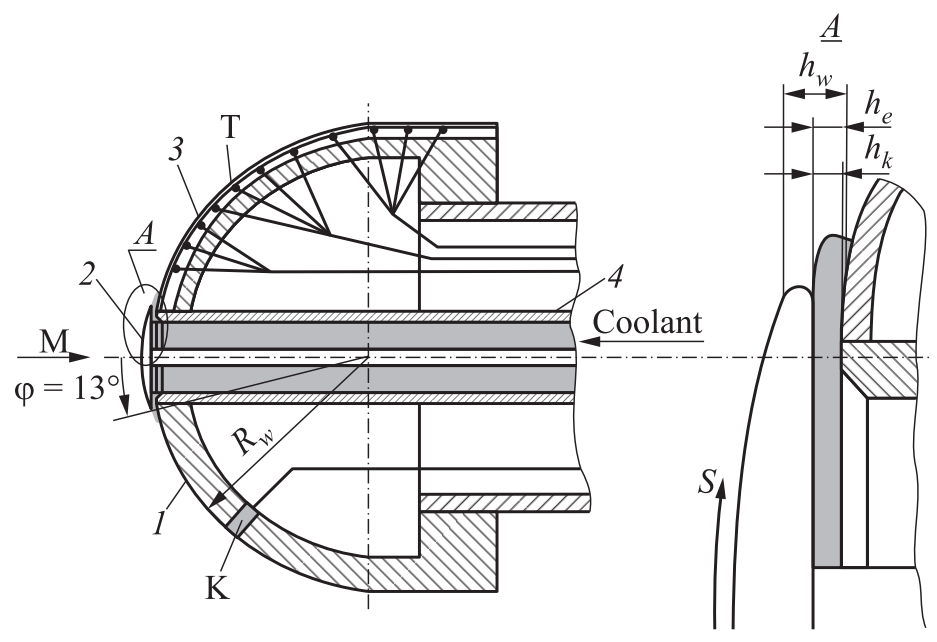

Figure 2 The model with the tangential slot on the spherical nosetip: 1 - nose; 2 - cup of slot; 3 - thin wall; 4 - coolant channel; $\mathrm{T}$ - thermocouples; $\mathrm{K}$ calorimeters; and $h_{k}-$ slot

system is much lower than the sound speed even in the case of maximum slot width $h_{k}=0.8 \mathrm{~mm}$. The tests of the supply system together with the model showed that hydraulic resistance of the system is less than $1 \%$ of the model resistance.

Duration of the hydraulic experiments essentially exceeded duration of tests in the wind tunnel. That is why, the error in injecting gas rate measurement did not exceed $3 \%$.

During experiments in the wind tunnel, the cooling gas supply was started prior to the diaphragm rupture with the advancing $\tau=0.1-0.5 \mathrm{~ms}$ and it was

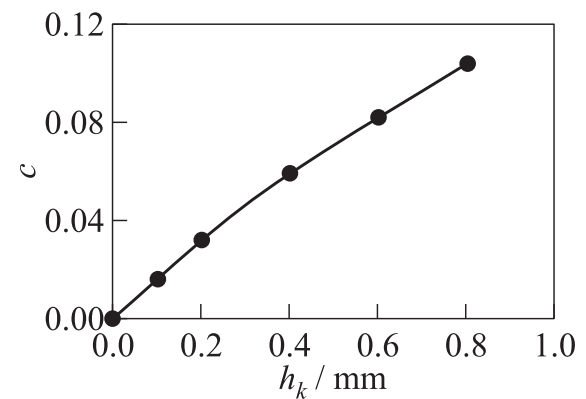

Figure 3 The hydraulic resistance of the slot $c\left(h_{k}\right)$ vs. the slot width $h_{k}$ stopped after the end of heat flux measurements. Pressure of injected gas was $P_{b}=2-32$ bar. The injecting mass rate was $G_{j}=0-4 \mathrm{~g} / \mathrm{s}\left(G_{j}\right.$ $=0$ at $\left.h_{c}=0\right)$ that corresponded to the dimensionless flow rate $G^{*}$ $=G_{j} /\left(\pi \rho_{\infty} u_{\infty} R_{w}^{2}\right)=0-0.12$ where $\rho_{\infty}$ and $u_{\infty}$ are the free stream density and velocity. Prior to the wind-tunnel experiments, careful measurements of injecting gas rate $G_{j}$ were carried out (Fig. 3 ) at various pressures $P_{b}$ in the vessel 18 


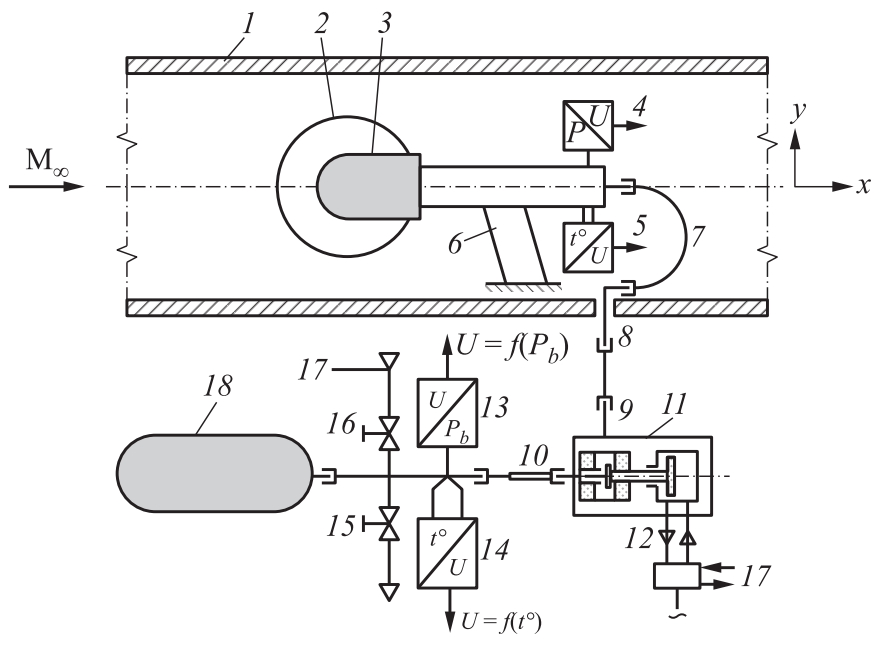

Figure 4 The supply system for gas injecting into the model: 1 - test section walls; 2 - optical window; 3 - blunt body; 4 - pressure gage at model inlet; 5 temperature gage at model inlet; 6 - pylon of model; $7-10$ - gas supply pipes; 11 main pneumatic-electrical valve; 12 - control electrical valve; 13 - vessel pressure gage; 14 - vessel exit temperature gage; 15 - vessel pressure release valve; 16 vessel filling valve; 17 - high-pressure tube; and 18 - vessel

(Fig. 4). It was found that $G=c\left(h_{k}\right) P_{b} / T_{b}^{0.5}$ where $T_{b}$ is the coolant temperature ahead the model and $c\left(h_{k}\right)$ is the experimentally determined dependence of the hydraulic resistance of the slot having the width $h_{k}$ (see Fig. 2).

The thin wall technique and calorimeters were used for measuring the heat flux to the body surface. A stainless steel foil band of 21-millimeter width and 0.2-millimeter thickness was welded to the model surface. From the inside, 0.1millimeter diameter kopel alloy wires were welded to the foil. Near the welding point, the wires were expanded to the thickness of $0.03-0.04 \mathrm{~mm}$. The dimensions of the thermocouple contact zone were $0.2 \times 0.2 \mathrm{~mm}$.

In accord with the thin wall technique, the heat flux was obtained using the one-dimensional heat conduction relation: $q=k d T / d \tau$ where $k \approx \rho c \delta$ is the calibration coefficient with $\rho$ and $c$ being the density and the heat capacity of the wall material and $\delta$ its thickness; $T$ is the temperature; and $\tau$ is the time. The coefficient $k$ is determined using the calibration device.

The effect of $2 \mathrm{D}$ heat conduction was investigated by numerical calculations and experimentally for the wall of different thicknesses $(\delta=0.1$ and $0.2 \mathrm{~mm})$. It has been found that the lateral heat conduction along the wall weakly affects the measurement results. 


\subsection{Supply System for Gas Injecting}

The coolant supply system is shown in Fig. 4.

The basic elements of this system are: the vessel 18 of about 40-liter capacity; the valve 16 for filling the vessel; the valve 15 for releasing pressure to atmosphere; the main pneumoelectric high-speed valve 11 .

The maximum gas pressure in the supply system was 150 bar. When main valve 10 is opened, the pressure in the model sets in approximately $0.2 \mathrm{~ms}$. Pressure $P_{j}$ and temperature $T_{j}$ in the gas supply system were registered at the vessel exit (gages 13 and 14, respectively) and at the model inlet (gages 4 and 5).

The pressure gage 4 has small inertia $(t \sim 2 \mathrm{~ms})$. Inertia of the temperature gage 5 is approximately the same as of the pressure gage.

\section{NUMERICAL PROBLEM FORMULATION AND SOLUTION METHOD}

The Navier-Stokes equations for axisymmetric $2 \mathrm{D}$ viscous compressible unsteady flows are solved numerically. The dimensionless conservative form of these equations is

$$
\frac{\partial \mathbf{Q}}{\partial t}+\frac{\partial \mathbf{E}}{\partial \xi}+\frac{\partial \mathbf{G}}{\partial \eta}=\mathbf{S}
$$

where $(\xi, \eta)$ is the curvilinear coordinate system; $x=x(\xi, \eta)$ and $r=r(\xi, \eta)$ are the Cartesian coordinates in a plane section of the cylindrical coordinate system; $\mathbf{Q}(\xi, \eta)$ is the vector of conservative variables; $\mathbf{E}(\xi, \eta)$ and $\mathbf{G}(\xi, \eta)$ are the flux vectors; and $\mathbf{S}(\xi, \eta)$ is the source vector. These vectors are expressed in terms of the corresponding vectors $\mathbf{Q}_{c}(x, r), \mathbf{E}_{c}(x, r), \mathbf{G}_{c}(x, r)$, and $\mathbf{S}_{c}(x, r)$ in the Cartesian coordinate system as

$$
\mathbf{Q}=J \mathbf{Q}_{c} ; \mathbf{E}=J\left(\mathbf{E}_{c} \frac{\partial \xi}{\partial x}+\mathbf{G}_{c} \frac{\partial \xi}{\partial r}\right) ; \mathbf{G}=J\left(\mathbf{E}_{c} \frac{\partial \eta}{\partial x}+\mathbf{G}_{c} \frac{\partial \eta}{\partial r}\right) ; \quad \mathbf{S}=J \mathbf{S}_{c}
$$

where $J=r \operatorname{det}[\partial(x, y) / \partial(\xi, \eta)]$ is the transformation Jacobian. Cartesian vector components for 2D Navier-Stokes equations are:

$$
\mathbf{E}_{c}=\left[\begin{array}{c}
\rho u \\
\rho u^{2}+p-\frac{\tau_{x x}}{\operatorname{Re}_{\infty}} \\
\rho u v-\frac{\tau_{x r}}{\operatorname{Re}_{\infty}} \\
\rho u H-\frac{1}{\operatorname{Re}_{\infty}}\left(u \tau_{x x}+v \tau_{x r}+\frac{\mu}{\operatorname{Pr}(\gamma-1) \mathrm{M}_{\infty}^{2}} \frac{\partial T}{\partial x}\right)
\end{array}\right] ;
$$




$$
\begin{gathered}
\mathbf{G}_{c}=\left[\begin{array}{c}
\rho v \\
\rho u v-\frac{\tau_{x r}}{\operatorname{Re}_{\infty}} \\
\rho v^{2}+p-\frac{\tau_{r r}}{\operatorname{Re}_{\infty}} \\
\rho v H-\frac{1}{\operatorname{Re}_{\infty}}\left(u \tau_{x r}+v \tau_{r r}+\frac{\mu}{\operatorname{Pr}(\gamma-1) \mathrm{M}_{\infty}^{2}} \frac{\partial T}{\partial r}\right)
\end{array}\right] ; \\
\mathbf{Q}_{c}=\left[\begin{array}{c}
\rho \\
\rho u \\
\rho v \\
e
\end{array}\right] ; \quad \mathbf{S}_{c}=\left[\begin{array}{c}
0 \\
\frac{1}{r}\left(p+\frac{\mu}{\operatorname{Re}_{\infty}}\left(\frac{2}{3} \operatorname{div} \mathbf{V}-2 \frac{v}{r}\right)\right) \\
0
\end{array}\right]
\end{gathered}
$$

Here, $e=p /(\gamma-1)+\rho\left(u^{2}+v^{2}\right) / 2$ is the total energy; $H=T /\left((\gamma-1) \mathrm{M}_{\infty}^{2}\right)$ $+\left(u^{2}+v^{2}\right) / 2$ is the total specific enthalpy; $\operatorname{div} \mathbf{V}=\partial u / \partial x+\partial v / \partial r+v / r$; and $\mathbf{t}$ is the stress tensor with components:

$$
\tau_{x x}=\mu\left(\frac{2}{3} \operatorname{div} \mathbf{V}-2 \frac{\partial u}{\partial x}\right) ; \tau_{x y}=\mu\left(\frac{\partial u}{\partial r}+\frac{\partial v}{\partial x}\right) ; \tau_{r r}=\mu\left(\frac{2}{3} \operatorname{div} \mathbf{V}-2 \frac{\partial v}{\partial r}\right)
$$

The fluid is a perfect gas with the specific heat ratio $\gamma=$ const and Prandtl number $\operatorname{Pr}=$ const. The system of equations is closed by the state equation

$$
p=\frac{\rho T}{\gamma \mathrm{M}_{\infty}^{2}} .
$$

The dynamic viscosity $\mu$ is calculated using Sutherland's formula

$$
\mu=\frac{T^{3 / 2}\left(T_{\mu}+1\right)}{T_{\mu}+T}
$$

where $T_{\mu}=110 \mathrm{~K} / T_{\infty}^{*}$. The second viscosity is assumed to be zero.

The dependent variables are normalized to the corresponding free-stream parameters: pressure - to the doubled dynamic pressure $\rho_{\infty}^{*} U_{\infty}^{* 2}$; the coordinates - to the reference length $L^{*}=1 \mathrm{~mm}$; and time $t-$ to $L^{*} / U_{\infty}^{*}$. The computations were carried out at the free-stream Mach number $\mathrm{M}_{\infty}=6$, the Reynolds number $\operatorname{Re}_{\infty}=L^{*} U_{\infty}^{*} \rho_{\infty}^{*} / \mu_{\infty}^{*}=2.0267 \cdot 10^{4}, \gamma=1.4, \operatorname{Pr}=0.72$, $T_{\infty}^{*}=86.33 \mathrm{~K}$, and $T_{w}^{*}=300 \mathrm{~K}$. These flow parameters correspond to the experimental conditions.

The Navier-Stokes equations are integrated numerically using an implicit finite-volume method with the second-order approximation in space and time. A quasi-monotonic Godunov-type scheme (TVD scheme) with Van-Leer limiter [16] are used. This gives a system of nonlinear algebraic equations, which is solved using the Newton iteration method. At each iteration step, the corre- 


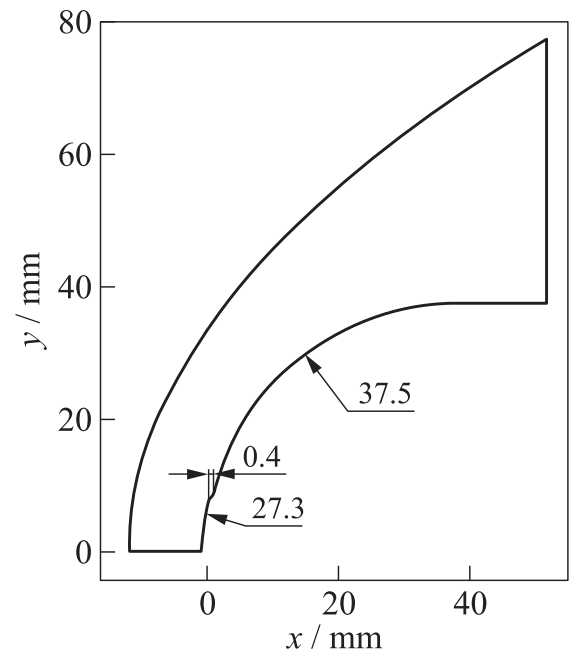

Figure 5 Computational domain

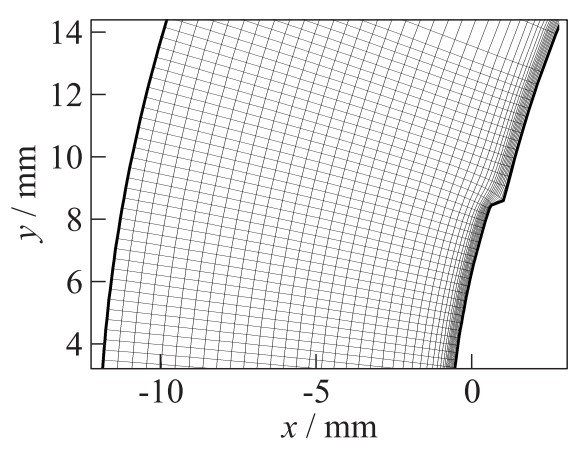

Figure 6 Grid near the injecting region (every 10 th line is shown) sponding linear system is solved by the GMRes (generalized minimal residual) method. Note that this approach is most efficient if the computational domain contains shock waves and other strong spatial inhomogeneities of a flow such as boundary-layer separation.

The boundary conditions are: no-slip conditions on the wall ( $u$ $=v=0)$; symmetry conditions on the axis in front of the body; free-stream conditions on the upper boundary $(u=1 ; v=0$; $p=1 /\left(\gamma \mathrm{M}_{\infty}^{2}\right)$; and $\left.T=1\right)$; and the linear extrapolation from the interior for the dependent variables $u$, $v, p$, and $T$ on the right (outflow) boundary $\left(f_{i}-2 f_{i-1}+f_{i-2}=0-\right.$ "soft" boundary conditions). The body surface is isothermal with temperature $T_{w}=3.47$. Numerical simulations require an additional condition on the wall pressure. This condition is obtained by extrapolation of the near-wall pressure to the surface assuming that $\partial p_{w} / \partial n=0$.

The computational domain is shown in Fig. 5. The generatrix of the upper boundary is parabola with the front point coordinates: $(-12 \mathrm{~mm}, 0 \mathrm{~mm})$ and the rear point coordinates: $(55 \mathrm{~mm}, 79.5 \mathrm{~mm})$. The body (and, therefore, the wall boundary) is composed of a hemisphere of radius $R_{w}=37.5 \mathrm{~mm}$ followed by a longitudinal cylinder. In front of the hemisphere, there is a "cap" with radius $r=27.3 \mathrm{~mm}$ and height of $25 \mathrm{~mm}$ stepped out by $h=0.4 \mathrm{~mm}$ from the main body.

Computations are performed on a curved orthogonal grid. It is generated using numerical conformal mapping of a rectangle onto the computational domain [17]. The grid is clustered near the surface so that $55 \%$ of the nodes are within the boundary layer or in the separation region with the mixing layer. The grid segment near the injecting region is shown in Fig. 6. 


\section{RESULTS OF INVESTIGATIONS}

\subsection{Numerical Results}

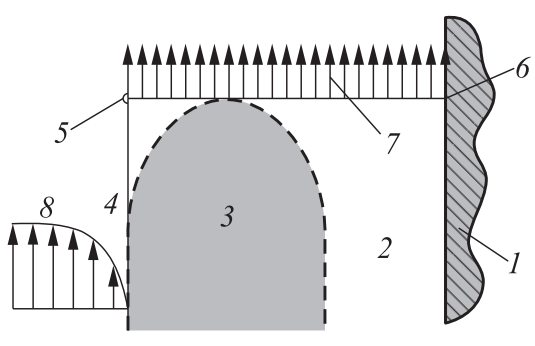

Figure 7 Injecting scheme: 1 - sphere; 2 - slot; 3 - "cap" edge; 4 - cap front surface; 5 and $6-$ boundaries of the injecting jet for numerical simulations; 7 injecting jet; and 8 - turned main flow
The flow is computed in the two regimes: with and without coolant injecting. The injecting gas parameters are: Mach number 2.53; pressure 15.2 bar; and temperature $273 \mathrm{~K}$. These parameters are set via the boundary conditions at the step between "cap" and hemisphere (Fig. 7).

The calculated flow temperature field is shown in Fig. $8 a$. It is seen that the separation zone is formed immediately downstream from the step between sphere and "cap." The separation bubble sizes agree well with the experimental results.

When gas is injected into the free stream, the flow field near the step is completely different (Fig. 8b).

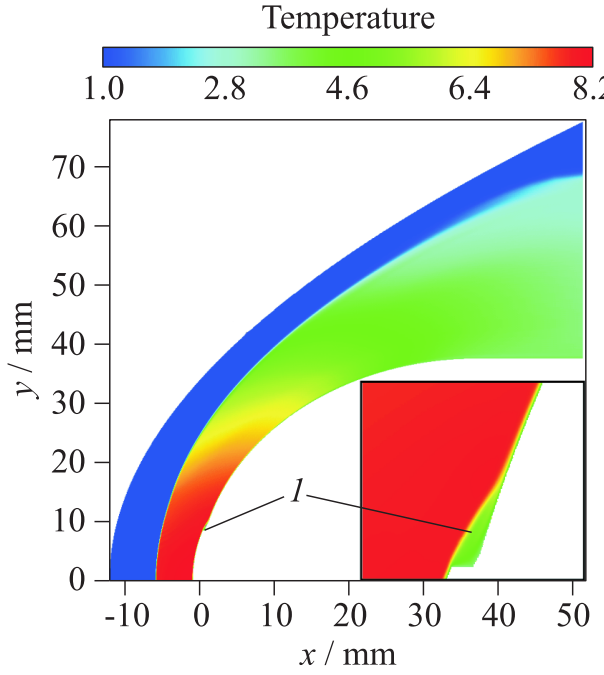

(a)

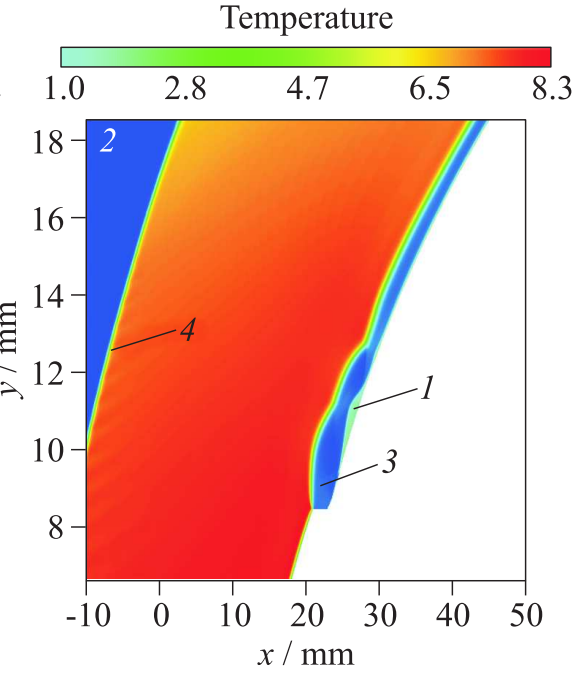

(b)

Figure 8 Temperature fields in symmetry plane without gas injecting $(a)$ and with injecting (mass rate $\left.G^{*}=0.072\right)(b): 1$ - separation zone; 2 - free flow; 3 - blowing jet; and 4 - bow shock 
The separation zone immediately downstream from the step is not observed, the injecting jet width notably increases with a distance from the slot. At a distance of 3 jet widths, the injecting jet separates from the sphere surface. Further downstream, the jet reattaches and then the cooling gas veil flows further without separation. Temperature of the cooling gas veil is lower than that of the near-wall main flow everywhere in the computational domain.

The density field of the main flow and the injecting jet is shown in Fig. 9. The zones of higher density formed in the shock waves are clearly seen in the injecting jet.
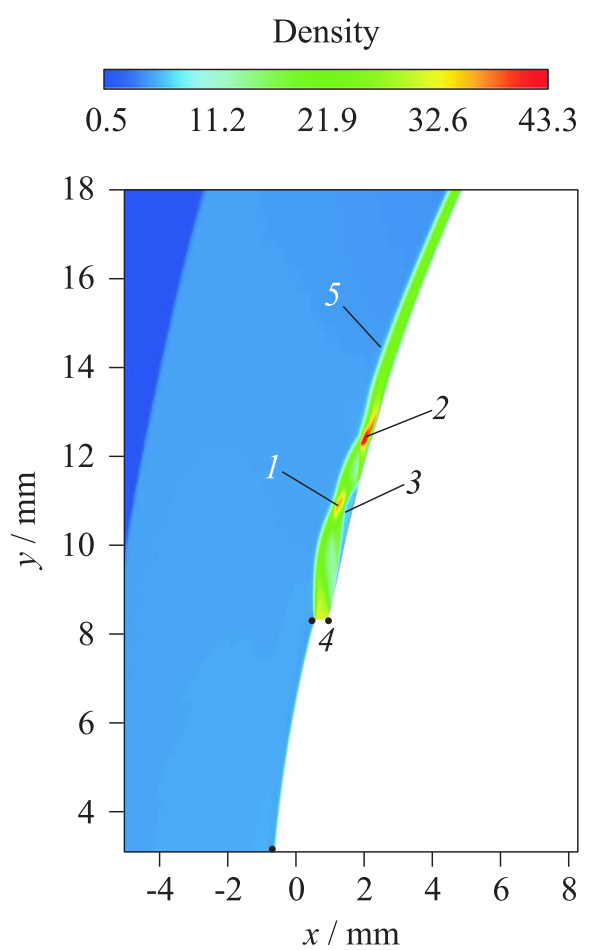

Figure 9 Density field in symmetry plane with injecting jet $G^{*}=0.072: 1-$ shock wave in injected flow; 2 - second shock; 3 - separation zone; 4 - boundary points of the slot outlet section; and 5 - mixing layer near wall flow
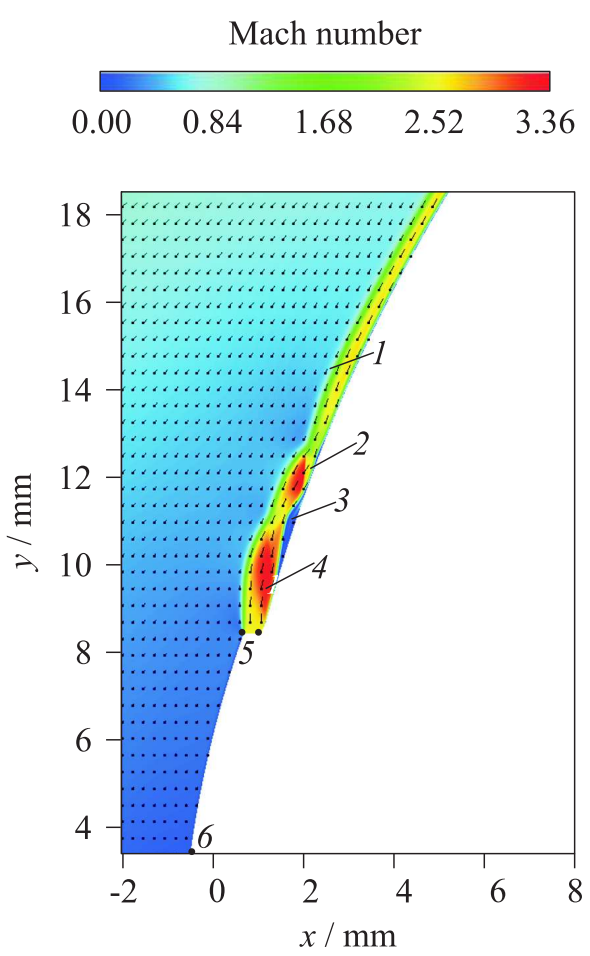

Figure 10 The Mach number field and the velocity vectors at the injecting mass rate $G^{*}=0.072: 1-$ mixing injected and near-wall flows; 2 - second separation zone; 3 - separation zone; 4 - shock wave in injected jet; 5 - boundary points of the slot outlet section; and 6 - critical point 
The velocity vectors in Fig. 10 show the presence of the reverse flow inside the separation zone.

The calculated heat flux is in qualitative agreement with the experimental data (Fig. 11a). However, the predicted heat flux is substantially lower than that measured in the experiment. The simplification (uniform speed at the output jet and the use of larger boundaries of jet than in an experiment) is an important reason for the differences between experimental and numerical data [18]. In the future work, the present authors will try to include the effects of nonuniform and nonstationary flow.

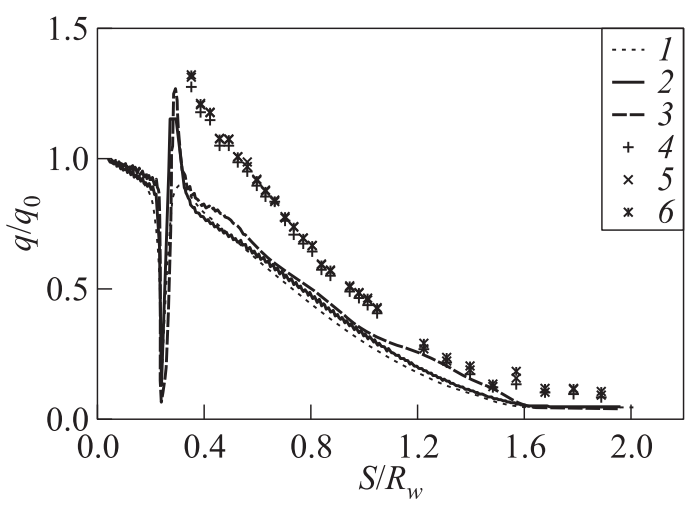

(a)

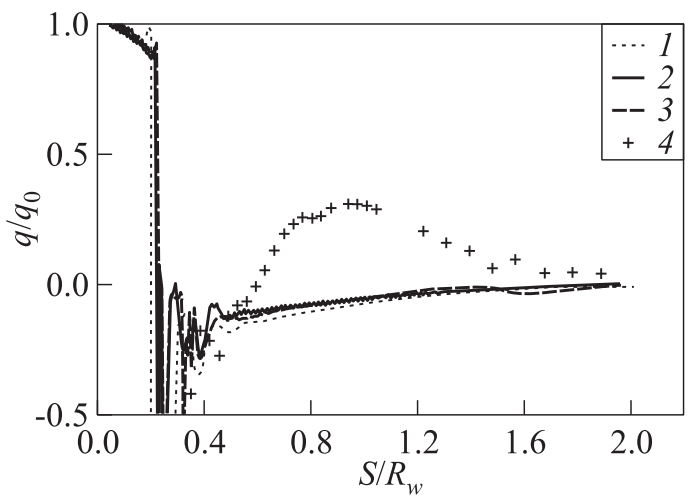

(b)

Figure 11 The heat-flux distributions on the sphere surface without injecting, $G^{*}$ $=0(a)$ and with injecting, $G^{*}=0.078(b): 1$ - calculation with grid $301 \times 201 ; 2-$ calculation with grid $601 \times 401 ; 3$ - calculation with grid $601 \times 401$ refined near the "cap" and slot; and 4-6 - experiments 
Figure $11 a$ shows also that the numerical solution does not depend on the grid fineness except for the heat-flux peak region where the coolant jet reattaches the surface. Finer grids are needed to simulate the flow in this region correctly.

The heat-flux distribution with injecting essentially differs from that of without injecting. The substantial decrease of the heat flux is observed (Fig. 11b) on the whole model surface (up to $S / R_{w}=1.9$ ).

\subsection{Experimental Results}

The heat fluxes obtained in the experiments are nondimensionalized by $q_{0}$ ratabled using the Fay's and Riddell's formula for the critical point on sphere [19]. Under the conditions of UT-1 wind tunnel (in presence of moisture, dust in free stream, and other reasons), the actual heat flux on the model can exceed this value. The excess level increase can reach $60 \%$. Therefore, at the further analysis, the relation of a thermal stream in the given point of a surface $q$ to the experimentally received maximum value of a thermal stream on a model surface in the critical point $q_{0 e}$ will be used.

The "cap" disturbs the spherical shape of the blunted nose. For the experiments with sealed slot, this can be seen on shadow patterns as deformation of the bow shock in front of the slot. On the other hand, the coolant injecting affects the efficient shape of bluntness. For moderate injecting rates, the shape is approximately spherical.

Therefore, the mentioned shock deformation can be appreciably smoothed (Fig. 12a). Note that with the flow rates used in the experiments, the effect of injecting on the shock-layer thickness is practically negligible. However, as it can be seen in shadow pattern, the cooling air jet introduces the disturbances into the flow over the model.

At the angle of attack of the model and the small expense of injected gas $P_{j 0}$, different flow pattern on windward generatrix of the model is possible. Momentum of injected gas can be insufficient for penetration into area of a stagnation point. Then, the basic flow forces the injected jet to change its direction toward the base of the model (Fig. 12b). Before the jet, there is a local shock wave $(A)$. Thus, the neighborhood of a critical point is not cooled.

In some runs without cooling, the slot was sealed by the rubber pad. Without sealing, the heat fluxes near the slot can be higher (approximately by 10\%) due to slight suction of hot air through the slot into the model. The results of the experiments with the sealed slot are shown in Fig. 13. The heat fluxes at some distance form the slot appreciably increase with the growth of the angle of attack. It is associated with a number of causes, in particular, with the displacement of critical point relative to the slot and with forming a separation zone behind it. 


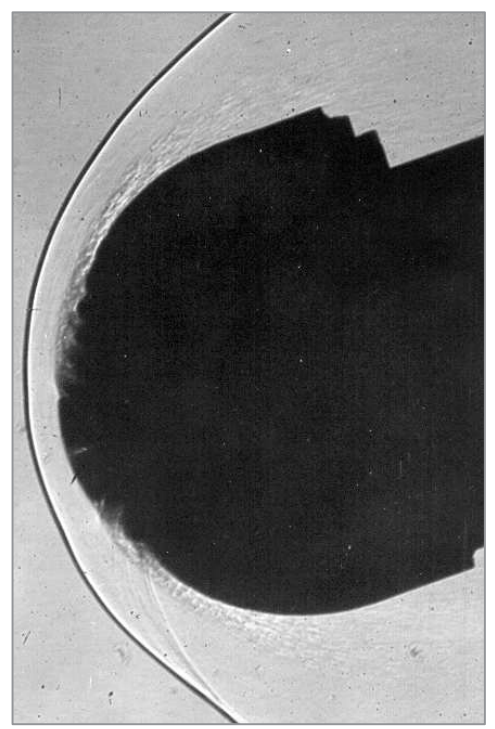

(a)

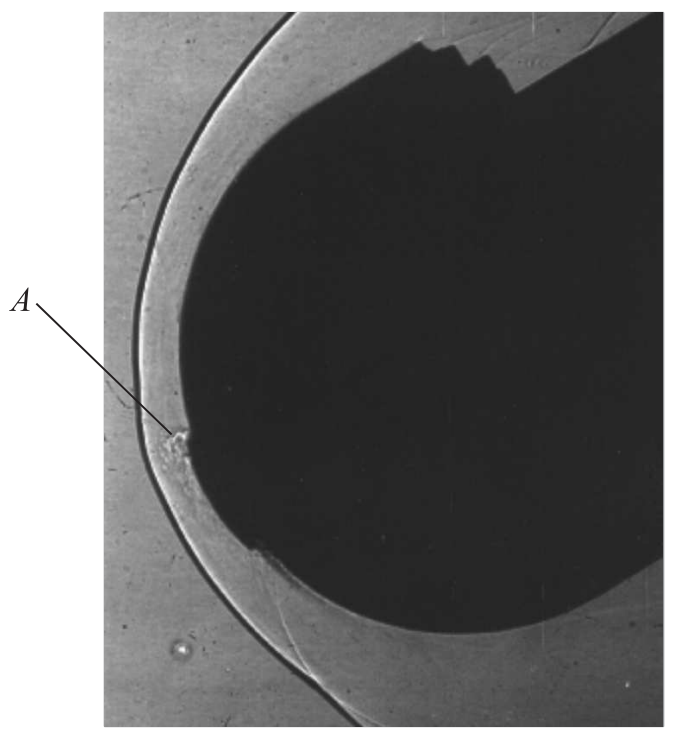

(b)

Figure 12 Shadow pattern of the flow around the model $\left(h_{k}=0.2 \mathrm{~mm}\left(h_{k}^{*}\right.\right.$ $\approx 0.0053)):(a) \alpha=15^{\circ}$ and $G^{*}=0.1556$; and $(b) \alpha=30^{\circ}, G^{*}=0.005, P_{s}=0.5$ bar, and $P_{j 0}=1$ bar

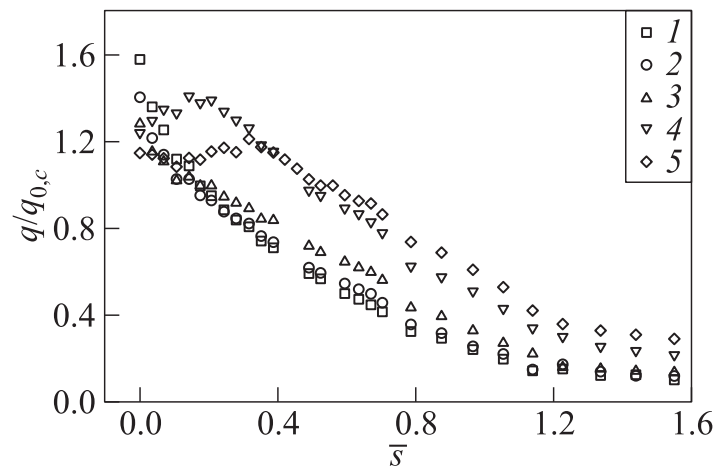

Figure 13 Effect of attack angle $\alpha$ on relative heat flux distribution $q=q / q_{0, c}$ along longitudinal coordinate $\bar{s}=s / R_{w}$ for the model with sealed slot: $1-\alpha=0^{\circ} ; 2-5^{\circ}$; $3-10^{\circ} ; 4-20^{\circ}$; and $5-\alpha=30^{\circ}$ 


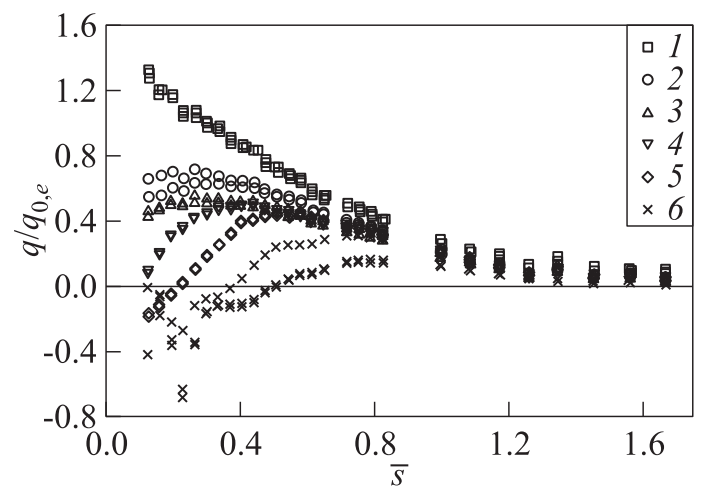

Figure 14 Effect of the angle of attack $\left(\alpha=0^{\circ}\right)$ and injecting on the relative heat flux distribution $q=q / q_{0, e}$ along the $\bar{s}$-coordinate; $h_{k}=0.2 \mathrm{~mm}$; windward: 1 $\bar{G}=0 ; 2-0.005 ; 3-0.010 ; 4-0.020 ; 5-0.078 ;$ and $6-\bar{G}=0.155$

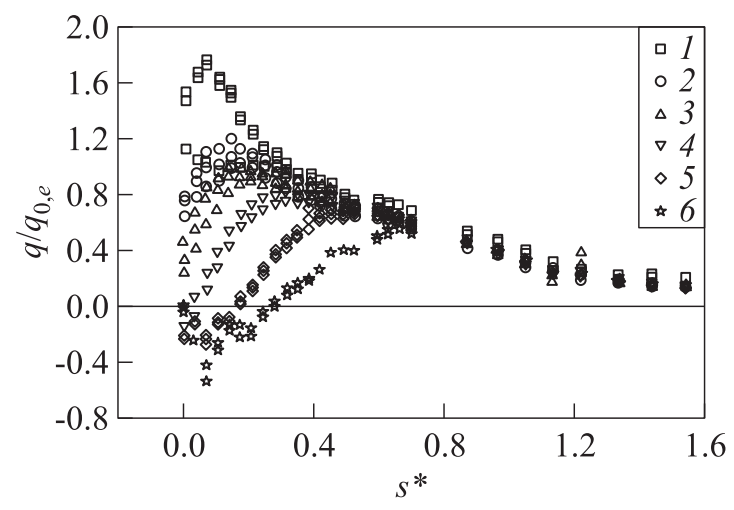

Figure 15 Effect of the angle of attack $\left(\alpha=20^{\circ}\right)$ and injecting on the relative heat flux distribution $q=q / q_{0, c}$ along the $\bar{s}$-coordinate (see Fig. 2 ); $h_{k}=0.2 \mathrm{~mm}$ $\left(h^{*}=0.0053\right): 1-\bar{G}=0 ; 1-0.0100 ; 3-0.0195 ; 4-0.0398 ; 5-0.0789$; and $6-0.157$

Downstream from this zone, the separated flow reattaches to the model surface that is accompanied by the heat flux growth.

The experiments with injecting showed that with the coolant mass rate being fixed, the relative heat flux is negligibly affected by the slot width. Therefore, in further consideration, only the effect of coolant mass rate $\bar{G}$ and angle of attack $\alpha$ on heat transfer rates are discussed (Figs. 14 and 15).

The injecting reduces the heat fluxes most in the case of zero angle of attack (see Fig. 14), when even for small mass rates (of order of $\bar{G}=0.005$ ), the peak 
value $\bar{q}_{m}=q_{m} / q_{0}$ is decreased by a factor of 2 and the peak moves away from the slot to the point $\bar{s}=s_{m} / R \approx 0.25$.

Qualitatively, the dependence $\bar{q}(\bar{G})$ remains the same for nonzero angles of attack (see Fig. 15). It is seen that when $\bar{G}$ is constant, the heat-flux peak location with respect to the slot slightly depends upon the angle of attack. However, the peak value increases that is an evidence of decreasing efficiency of the injecting. For instance, at $\bar{G}=0.157$, the maximum heat flux

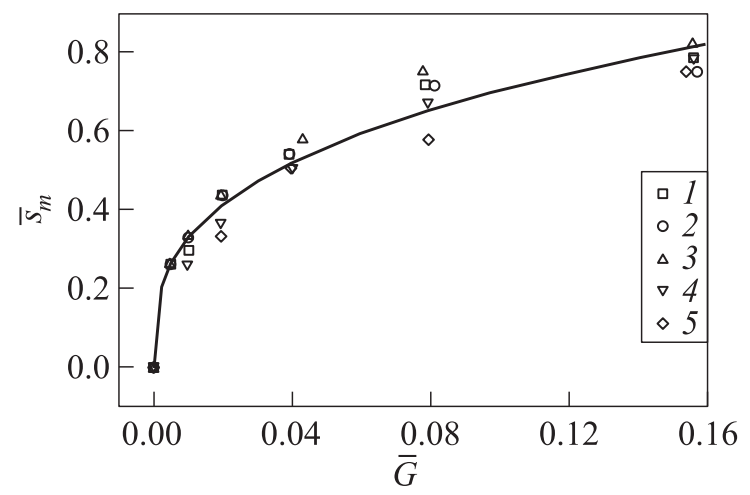

Figure 16 Dependence of $\bar{s}_{m}$ on dimensionless injecting rate $\bar{G}$; signs refer to experimental data and curve to approximation on equation $\bar{s}_{m} \approx 1.5 \bar{G}^{0.33} ; h=0.2 \mathrm{~mm}$ : $1-\alpha=0^{\circ} ; 2-5^{\circ} ; 3-10^{\circ} ; 4-20^{\circ}$; and $5-\alpha=30^{\circ}$

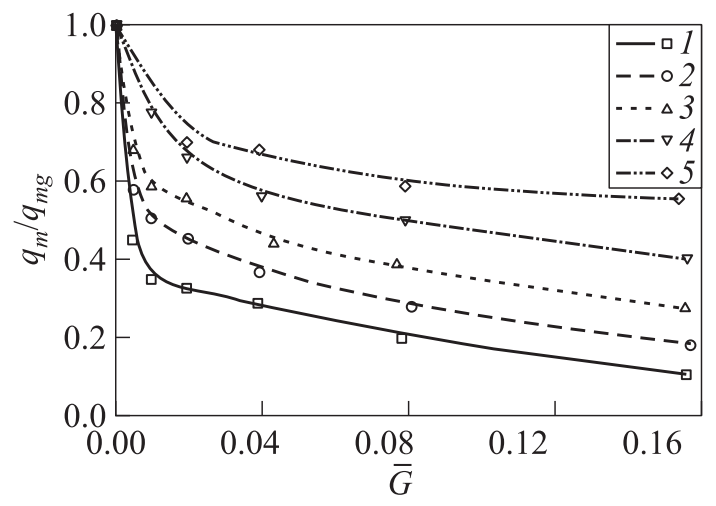

Figure 17 Effect of the dimensionless injecting rate $\bar{G}$ and angle of attack on the relative heat flux maximum $q_{m} / q_{m g} ; h_{k}=0.2 \mathrm{~mm}: 1-\alpha=0^{\circ} ; 2-5^{\circ} ; 3-10^{\circ}$; $4-20^{\circ}$; and $5-\alpha=30^{\circ}$ 
is reduced by a factor of 2.5 rather than by a factor of 10 in the case of $\alpha=0^{\circ}$.

According with the foregoing discussion, the heat-flux peak location is driven primarily by the coolant mass rate.

This conclusion is confirmed by the experimental data shown in Fig. 16 in terms of $\bar{s}_{m}(\bar{G})$ for all angles of attack considered. The curve shows the approximate dependence.

The dependence of maximal heat flux $q_{m}$ on the injecting rate is shown in Fig. 17 for different angles of attack. It is normalized by the maximal heat flux $q_{m g}$ on the model with the sealed slot. For $\bar{G}>0$, the effect of the angle of attack on the relative maximal heat flux can be estimated using the relation

$$
\frac{q_{m}}{q_{m g}} \approx\left(\frac{q_{m}}{q_{m g}}\right)_{n}+0.013 \alpha
$$

where $\left(q_{m} / q_{m g}\right)_{n}$ is the relative heat flux at zero angle of attack.

\section{CONCLUDING REMARKS}

The tangential gas blowing through an axisymmetric slot located near the critical point of a spherically blunted cylinder leads to decreasing of the heat flux on the blunted nose and the adjacent cylindrical part.

The heat flux reduction is attained in the range of angles of attack from $0^{\circ}$ to $30^{\circ}$. However, at high angles of attack, essentially greater coolant flow rate is needed compared to the case of zero angle of attack.

The main parameter governing the heat flux decrease appears to be the mass rates ratio $G^{*}$ of the blowing matter and free flow.

The shape of the bow shock in front of the blunted nose does not practically depend on $G^{*}$.

Numerical simulations provide insight into the flow-field structures of the main flow and the blowing gas. The predicted heat-flux distribution qualitatively agrees with the experimental results. However, turbulence should be accounted for to get a good quantitative agreement.

\section{ACKNOWLEDGMENTS}

Subsection 4.1 of this work was carried out in the Moscow Institute of Physics and Technology under the financial support of the Russian Science Foundation (project No.14-19-00821). Subsection 4.2 of this work was supported by the Russian Foundation for Basic Research (project No. 14-01-00971). 


\section{REFERENCES}

1. Vasilevsky, E. B., V. Ya. Borovoy, and M. N. Osin. 1992. Optical methods for the investigation of the fluid blowing into a supersonic air flow. Conference (International) on the Methods of Aerophysical Research (ICMAR) Proceedings. Novosibirsk, Russia. II:15-18.

2. Vasilevsky, E. B. 1994. Active thermal protection. Research in Hypersonic Flows and Hypersonic Technologies: TsAGI Workshop-School "Fluid Mechanics." Zhukovsky, Russia. 28-32.

3. Vasilevsky, E. B. 2003. Behavior of liquid sprayed through a centrifugal injector against supersonic gas stream. Conference (International) on Fundamental Research in Aerospace Science Proceedings. Zhukovsky, Russia. 4:11-13.

4. Vasilevsky, E. B. 2003. Teplozashchita poverkhnosti tela ot konvektivnogo teplovogo potoka putem vduva [Convective heat flux protection of a surface by means of blowing]. Aeromechanics Gasdynamics 2:37-48.

5. Stepanov, E. A., V.N. Kharchenko, and Z.S. Ogorodnikova. 1973. Techenie gaza s massoobmenom na poverkhnosti [Gas flow with mass transfer on a surface]. TsAGI Scientific-and-Engineering Information Department. Review No. 436. $259 \mathrm{p}$.

6. Feldhuhn, R. H. 1976. Heat transfer from a turbulent boundary layer on a porous hemisphere. AIAA Paper No. 76-119.

7. Holden, M. S., K. M. Rodriges, and B. J. Nowak. 1991. Studies of shock/shock interaction on smooth and transpiration-cooled hemispherical nosetips in hypersonic flow. AIAA Paper No. 91-1765.

8. Foster, L. E. 2010. Transverse blowing into subsonic crossflow with various injector orifice geometries. AIAA Paper No. 2010-55.

9. Maddalena, L., and P. E. Dimotakis. 2010. On the effects of transverse-jet blowing into a supersonic shear layer. AIAA Paper No. 2010-755.

10. Borovoy, V. Ya., Ed. B. Vasilevskii, I. V. Struminskaya, and L. V. Yakovleva. 1998. Gas flow and heat protection by strong blowing in the shock wave interference region near the blunt body front surface. 3rd European Symposium on Aerothermodynamics for Space Vehicles Proceedings. 301-307.

11. Vasilevskiy, E. B., and L. V. Yakovleva. 2009. The tangential gas blowing as the means of heat protection of a blunt body streamlined by dusted gas. European Conferences for AeroSpace Sciences (EUCASS 2009).

12. Heufer, K. A., and H. Olivier. 2008. Experimental and numerical study of cooling gas blowing in laminar supersonic flow. AIAA J. 46(11):2741-2751.

13. Hombsch, M., and H. Olivier. 2010. Flow condition and cooling gas variation for film cooling studies in hypersonic flow. Sonderforschungsbereich/Transregio 40. Annual Report. 27-39.

14. Hombsch, M., and H. Olivier. 2011. Film cooling in turbulent supersonic flow. Sonderforschungsbereich/Transregio 40. Annual Report. 19-29.

15. Czajkowski, E. 1994. Russian aeronautical test facilities. Arlington, VA: ANSER Center for International Aerospace Cooperation. 
16. Egorov, I. V., A. V. Fedorov, and V. G. Soudakov. 2006. Direct numerical simulation of disturbances generated by periodic suction-blowing in a hypersonic boundary layer. Theor. Comp. Fluid Dyn. 20(1):41-54.

17. Driscoll, T. A., and S. A. Vavasis. 1998. Numerical conformal mapping using crossratios and Delaunay triangulation. SIAM J. Sci. Comput. 19(6):1783-1803.

18. Bezmenov, V. Ya., V. Ya. Borovoy, I. V. Struminskaya, and L. V. Yakovleva. 1999. Influence of disturbances generated by the nozzle boundary layer on the heat exchange on the cylindrical nose of a body in a hypersonic flow. Fluid Dyn. 34(1):105109.

19. Fay, J.A., and F.R. Riddell. 1958. Theory of stagnation point heat transfer in dissociated air. J. Aeronaut. Sci. 2:73-85. 